\title{
In the Era of Digitalising Shop Floor Management: In Blissful Ignorance of Superfluous Work
}

\author{
John Bang MATHIASEN ${ }^{1}$ and Henning de HAAS \\ Aarhus University, Department of Business Development and Technology
}

\begin{abstract}
This study aims to understand the extent of superfluous work at shop floors and suggests some managerial opportunities for reducing superfluous work. Drawing on the abductive reasoning, the research systematically combines a theoretical conceptualisation of decision-making processes in a digitalised manufacturing with an empirical enquiry of a smart manufacturing. The paper reveals superfluous work if decision-making processes cross disciplinary and/or organisational boundaries. Superfluous work occurs because of lacking data and information to guide reflective thinking and knowledge sharing. In relation to high complex decision making the ongoing implementation of workarounds does also cause superfluous work. Prerequisites for reducing superfluous work are accessibility of applicable data to guide reflective thinking and knowledge sharing.
\end{abstract}

Keywords. Shop Floor Management, decision-making, smart manufacturing, superfluous work, knowledge sharing

\section{Introduction}

Companies witness an ongoing transition towards smart manufacturing [1], which makes Shop Floor Management (SFM) decision-making topical. SFM decision making is pivotal for an efficient execution of manufacturing [2], but to what extent if any does decision making in a smart manufacturing influence the amount of superfluous work?

Superfluous is defined in Cambridge English Dictionary as "more than is needed; extra and not necessary". Superfluous work is often considered as value adding by practitioners carrying out the work, but basically it does not add value [3]. Superfluous work is hidden waste [4]. Superfluous work occurs because of practitioners lack data and/or information to accomplish the decision-making process. In this research superfluous work equals the gap between necessary work and actual work spent on accomplishing decision-making. Necessary work is the minimum work if having access to reliable data and can apply this; actual work = necessary work + superfluous work.

In a smart manufacturing set-up the complexity of decision-making varies [5]. Straightforward decision making is handled within disciplinary boundaries, but complex issues call for collaboration across disciplinary and/or organisational boundaries [6]. Wognum et al. [7] agree and argue that the development and implementation of smart manufacturing calls for transcending disciplinary boundaries, mainly due to both social and technical issues are ill-defined and thus characterised by high complexity.

\footnotetext{
${ }^{1}$ Corresponding Author, Mail; John Bang Mathiasen, johnbm@btech.au.dk.
} 
In the current era of digitalising manufacturing equipment and information systems, SFM decision making draws still on principles developed in mid-50s [8] and applied information systems appear as they did 20-30 years ago [9]. Given that digitalised equipment enabling data-driven decision-making has not yet entered shop floors, SFM is most likely characterised by superfluous work. It seems managers in companies live in blissful ignorance of these consequences.

To gain an understanding of SFM decision making in smart manufacturing set-ups this study aims to understand the extent of superfluous decision-making work and suggests some managerial opportunities for reducing the amount of superfluous work. The following two research questions guide the study "to what extent does decision-making complexity influence the amount of superfluous work?" and "what are the prerequisites for reducing superfluous work?". To answer these two research question the study draws upon three cases from a company operating globally. The three cases explicate the characteristics of SFM decision making; one case addresses decision making within disciplinary boundaries, and two cases elaborate decision making across disciplinary/organisational boundaries. By juxtaposing the empirical findings from the three cases with related theories, we conceptualise a framework with the purpose of clarifying the consequences of and suggestions for reducing superfluous work.

The contributions are as follows: Low decision making complexity does not lead to superfluous work. Medium decision-making complexity is protracted and superfluous work occurs in relation to gain access to applicable data and information. Handling highly complex issues are a lengthy process back and forth disciplinary/organisational boundaries, yet the extent of superfluous work is mainly related to implementing workarounds. Prerequisites for reducing superfluous work are accessibility of applicable data to guide reflective thinking and knowledge sharing.

\section{Theory}

A digitalised shop floor is an arrangement of digitalised manufacturing equipment and information systems to manage employees and optimise the flows of materials and information. Some researchers conceptualise a digitalised shop floor as a Cyber Physical Systems $[10 ; 11]$ and as data analytics practices [12], which enables information transparency [13] and high utilisation of resources [2; 14].

The industrial rethinking represented by Industry 4.0 increases the complexity of decision-making processes, the diversity of data and the pace of changes to be handled $[15 ; 16]$. Despite the increasing complexities it is expected that machine learning algorithms [17], interoperability [10;18] and artificial intelligence [19] lead to a situation where decision-making processes are accomplished by digital technologies. Implicitly, this stream of research gives technologies deterministic effects, meaning that decision making will be automatised and thus handled without the involvement of practitioners. However, it is implausible that machine learning, algorithms and artificial intelligence will exceed practitioners' creative intelligence, social intelligence, intuition and judgement in terms of decision making. Thus, in the future decision making involves both digital technologies and practitioners' knowledge [8]. SFM decision making is a process of applying accessible data and information with the purpose of eliminating waste.

Superfluous work is a kind of hidden waste. Lean and elimination of waste go hand in hand [20]. Waste is often categorised into different types of waste, which are unnecessary transportation, defects, overproduction, waiting, excess inventory, unnecessary 
movement, incorrect processing and underutilisation of people and their creative input for improvements [21]. Sutrisno et al. [22] suggest four digital waste types, which are i) obsolete data and information, ii) low accessibility of information, iii) scattered information thus lack of shared understanding, and finally iiii) inappropriate coordination.

While the waste factors suggested by Womack and Jones [21] are directly perceptible for the involved practitioners, the four types of factors proposed by Sutrisno et al. [22] are hidden waste. Decision making based on these hidden waste factors - obsolete information, low accessibility of information, scattered information across disciplinary boundaries and thus lack of shared understanding and finally inappropriate coordination across disciplinary boundaries will often result in slow running or idling equipment, waiting time, rush orders, overtime, expensive transportations, unnecessary movement of materials and rescheduling [21]. In addition, the hidden waste factors lead to superfluous work [2; 4], which means that practitioners carry out work they consider as being valuable, but basically it does not add any real value for the company. The hidden waste factors and thus the seed for superfluous work originate from low accessibility and applicability of data and information.

"Without data, you're just another person with an opinion" (W. Edwards Deming). This old saying highlights the importance of having access to data, which however is insufficient to carry out SFM decision making at digitalised shop floors. Rather, SFM decision making is enabled by the applicability of accessible data and information. In other words, data-driven decision making, accessibility and applicability are inseparable.

A smart manufacturing set-up causes increasing diversity of data [12]. The diversity of data are categorised as structured-, semi-structured- and unstructured data [13]. This combined with a general lack of syntactic-, semantic- and cross-domain interoperability [18] forms isolated information islands [23] and thus low accessibility of data and information. Information islands obstruct practitioners in gaining access to and in applying data to accomplish data-driven decision-making. The low information transparency causes to isolated decision-making.

Required data for enhancing decision-making responsiveness depends on the complexity of the problem and whether or not, the involved practitioners are capable of applying the accessible data to identify and implement a solution. Data-driven decision making is a process of converting data and information into embodied knowledge and knowledge sharing within and across disciplinary boundaries [6]. Given that the applicability of data and data-driven decision-making are inseparable, the value of "big data" depends solely on the information and knowledge, which can be derived from the data [24].

SFM decision-making and knowledge sharing unfold in symbiosis, which require practitioners to carry out reflective thinking [25]. Reflective thinking is an inquiry process [26] in which each of the involved practitioners' decision-making actions are "guided" by observations of cues from the surroundings and his/her experience [27]. These cues are malfunctions at the shop floor level, other practitioners' actions as well as accessible data and information. Experience is embodied and situational and according to Dewey [28] to activate reflective thinking, the cues from the surroundings must cause disturbance in the habitual way of decision making. This highlights the importance of the old saying "well begun is half done" and thus conceptualises decision making as a progressive inquiry process of the conditions at the shop floor. SFM decision making involves ongoing interplay between each practitioner's experience and observations; as for the latter, interpretation of social and technical conditions within the particular shop floor. Thus, the accessibility and applicability of data and information should be tailored to the complexity of the decision-making processes. 
SFM decision making involves both technical and social matters $[8 ; 29 ; 30]$. The definition of decision-making complexity is not unequivocal. Anderson and Törnberg [29] distinguish between complex and complicated issues. Complex issues deal with social matters as interactions in social network, which involves high redundancy, unpredictability and amplification of disturbances. The focal point for complicated issues is technical matters. It focuses on issues related to technologies consisting of scaleseparated level of hierarchies, predictability and decomposability. By combining complex and complicated issues three groups of decision-making processes are identifies; simple, sub-wicked and wicked decision making.

Researchers argue $[7 ; 12 ; 29]$ that wicked problems are ill-defined problems, which solution requires transcending professional disciplines. Drawing on this understanding and the work of Liker and Meyer [20] and Hertle et al. [5], Mathiasen and Clausen [6] suggest that; i) low complexity decision-making is handled within disciplinary boundaries of shop floors; ii) medium complexity issues necessitates interdisciplinary decision-making cutting across disciplinary boundaries; iii) high complexity decisionmaking requires transcending disciplinary boundaries.

\section{Methodological considerations}

This explorative study aims to understand the extent of superfluous decision-making work and suggests some managerial opportunities for reducing the amount of superfluous work. Accordingly, the paper follows Yin's [31] advices to use a case research method; a single case with three embedded cases are selected. The logic of inquiry is abductive and draws on Dubois and Gadde's [32] systematic combining, who suggest concurrent elaboration of the theoretical conceptualisation, the processing of data, the drawing up the cases and the analyses.

In an explorative study the learning opportunities from the empirical material is pivotal [33]. The selected case company has recently gone through a transformation towards a smart manufacturing set-up. The criterion for selecting the three embedded cases deals with opportunity to learn [33]. Diversity in terms of complexity of decision making is the criterion for selecting the three cases.

The data collection consists of observations, interviews and second-hand information. Regarding the observations, one of the authors has regularly visit the case company before, during and after the transition to the smart manufacturing set-up. Being present at the shop floors and taking the role of "complete observer" (see [34]) gives an understanding of decision-making processes, abbreviations and expressions applied by the practitioners. Likewise, it paves the way for several unstructured interviews. In addition to many unstructured interviews, two semi-structured interviews are conducted with the plant manager. Due to confidential reasons, the semi-structured are not taped. Instead, notes are taken and just after each interview notes are typed up and reflections from the interviews are added to the document. Final, both authors have many years of experience from working in manufacturing companies.

By systematically combining our theoretical and empirical understanding, the collected data are coded; i.e., decision-making complexity, accessibility and applicability of data and information and the consequences at the shop floor. Based on this coding, each of the three cases is analysed separately to expose the extent of superfluous decision-making work including waste. A cross-case analysis juxtapose the findings with the purpose of revealing managerial opportunities for eliminating superfluous work. 


\section{Cases}

The case factory, pseudo-named "Factory", is part of a global conglomerate. Factory is located in a high-wage areas and consequently capacity utilization and efficiency are fore fronted. Factory has been operating within the Fast-Moving Consumer Goods industry for several decades and has a good reputation in terms of delivering on time.

Factory has designed and constructed a completely new factory and subsequently all assembly lines have been renewed and highly automated. Currently, Factory has the highest degree of automation and digitalisation of manufacturing equipment in the conglomerate. The assembly lines are fully automated and various sensors along the lines collect performance data automatically, which ensures a reliable and appropriate data foundation. Accordingly, Factory has implemented an on-line system for monitoring and following up on Overall Equipment Efficiency (OEE).

Material handling at the end of the assembly lines is fully automated. After each of the assembly lines a conveyor belt transports the finish goods to the material handling department, where robots handle the palletising and data registration. The data registration requires retrieving and storing production and customer related data in the ERP systems, printing and fitting on barcodes, and inventory registration. The data registration involves several IT-systems, which means the interfaces among the IT-systems are very complex. The development and implementation of the material handling systems have required across discipline and organisational boundary collaboration.

In line with the other factories in the global conglomerate, Factory follows the lean principles for performance management and continues improvement. The OEE system is a useful monitoring tool and Factory reports weekly OEE performance to top management. However, the OEE application is a stand-alone system, which is neither integrated with the Enterprise Resource Planning (ERP) systems nor other IT systems being applied in the conglomerate. This lack of interfaces imposes complexity in term of using the accessible information to visualise and analyse different types of data; for instance OEE performance, delivery performance, maintenance and quality. According to the plant manager, approximate $60 \%$ of the collected data are used. In this regard two problems are put forward; i) practitioners do not have sufficient knowledge to interpret and thus use the data to identify the root-cause of the problem; ii) despite data are collected automatically by sensors, practitioners operating the assembly lines have to manually enter error codes, which has a negative impact on the reliability of the data stored in the IT-systems.

To facilitate knowledge sharing within each department and at the plant level three different board (kaizen) meetings are conducted; i) assembly line board meeting in which blue-collar workers, technicians and departmental manager participate; ii) departmental board meeting across the assembly lines having the purpose of coordination and identifying problematic issues, where the participants are the departmental manager and few appointed blue-collar workers and technicians; iii) plant board meeting discussing main issues across departments and assembly lines, participants are plant manager, departmental managers and lean manager. In general, decision making within departmental boundaries are handle promptly, but knowledge sharing involving practitioners from other department, factories or external business partners are a protracted process. In the following the three decision-making cases are briefly presented in table 1; these make up the empirical foundation for the following analytical chapter. 
Table 1. Three decision-making cases.

\begin{tabular}{|c|c|c|c|}
\hline Problematic issue & Knowledge sharing & Consequences & Duration \\
\hline \multicolumn{4}{|c|}{ Low complexity decision making } \\
\hline $\begin{array}{l}\text { Weight issues on } \\
\text { product produces }\end{array}$ & $\begin{array}{l}\text { Ongoing weight measuring characterised by } \\
\text { high visibility are directly understandable }\end{array}$ & High scrap, reduced OEE & $1 / 2$ hours \\
\hline $\begin{array}{l}\text { Infeed of material } \\
\text { to assembly line } \\
\text { not running }\end{array}$ & $\begin{array}{l}\text { High accessibility of structured data, which } \\
\text { are useable for practitioners enables } \\
\text { knowledge sharing within shop floor }\end{array}$ & $\begin{array}{l}\text { Downtime - idling and/or } \\
\text { slow running equipment. } \\
\text { Reduced OEE }\end{array}$ & Three hours \\
\hline $\begin{array}{l}\text { Incorrect filling of } \\
\text { products }\end{array}$ & $\begin{array}{l}\text { Only downtime data is displayed/available, } \\
\text { but the technicians have experience with } \\
\text { handling the problem }\end{array}$ & $\begin{array}{l}\text { Downtime - idling and/or } \\
\text { slow running equipment, } \\
\text { reduced OEE }\end{array}$ & Five hours \\
\hline \multicolumn{4}{|c|}{ Medium complexity decision making } \\
\hline $\begin{array}{l}\text { Software error } \\
\text { OEM equipment }\end{array}$ & $\begin{array}{l}\text { Only downtime data is displayed/available. } \\
\text { Software related data are only available for } \\
\text { supplier. Supplier can access and use data }\end{array}$ & $\begin{array}{l}\text { Downtime - idling and/ or } \\
\text { slow running equipment. } \\
\text { Reduced OEE }\end{array}$ & Two months \\
\hline $\begin{array}{l}\text { Serial defect in } \\
\text { inbound materials }\end{array}$ & $\begin{array}{l}\text { Factory lacks data - only approved product } \\
\text { specification data are available. Serial defect } \\
\text { data are forwarded to purchasing/suppliers }\end{array}$ & $\begin{array}{l}\text { Downtime and scrap of } \\
\text { material. Postponement of } \\
\text { deliveries. Reduced OEE }\end{array}$ & $\begin{array}{l}\text { Several } \\
\text { months }\end{array}$ \\
\hline $\begin{array}{l}\text { Software } \\
\text { interfaces }\end{array}$ & $\begin{array}{l}\text { Only OEE data available for Factory, limit } \\
\text { data of Software. Solutions implemented by } \\
\text { external partner }\end{array}$ & $\begin{array}{l}\text { Slow running equipment. } \\
\text { Reduced OEE }\end{array}$ & Four months \\
\hline \multicolumn{4}{|c|}{ High complexity decision making } \\
\hline $\begin{array}{l}\text { Low robustness of } \\
\text { technical solutions }\end{array}$ & $\begin{array}{l}\text { No data accessible and neither suppliers nor } \\
\text { Factory knows the solution }\end{array}$ & $\begin{array}{l}\text { Recurrent slow running } \\
\text { equipment. Reduced OEE }\end{array}$ & One year \\
\hline $\begin{array}{l}\text { Low dependability } \\
\text { of suppliers }\end{array}$ & $\begin{array}{l}\text { Delivery performance data are available, but } \\
\text { these data are not useful to enhance } \\
\text { dependability }\end{array}$ & $\begin{array}{l}\text { Recurrent stock-out of } \\
\text { inbound material - } \\
\text { planned downtime }\end{array}$ & Several years \\
\hline
\end{tabular}

\section{Analysis}

This research sets out to explore the extent to which decision-making complexity influence the amount of superfluous work?" and "the prerequisites for reducing superfluous work?".

As illustrated in the three cases, low complexity decision making occurs either directly at the assembly line or at one of the three board meetings. Low complexity decision making represents the majority of problematic issues being handled at shop floor level. The consequences are minor due to the fact that the duration from detecting to handling the abnormal situation is short. At the shop floor scrap of material, idling and slow running equipment are the cues for the occurrence of an abnormal situation. Too high scrape of material and reduced OEE are the cues at the board meetings. This kind of cues are sufficient to trigger the practitioners' reflective thinking and knowledge sharing. In general the required data and information are accessible from various standalone IT-systems. In particular, structured OEE data combined with the Lean A3 way of structuring the decision-making processes is applied to ensure that problems are handled promptly without any delays. In other words, the amount of superfluous work is minimal.

The consequences of medium complexity issues are perceptible at the shop floor. Cues highlighting the problematic situations are similar to low complexity decision making; at shop floor these are scrap of material, idling and/or slow running equipment, and at board meetings the cues are in general too high scrap and reduced OEE. Despite the daily consequences are equal to low complexity issues, the duration of operating with increased scrape and low OEE is rather long-term - from two to four months. The decision-making process is protracted. Cues from the physical shop floor - high scrap, idling or slow running equipment - or OEE/scrap data and information visualised at one 
of the three board meetings trigger the decision-making process. Either lack of experience with the particular problematic situation or low accessibility of structured, semi-structured and unstructured data/information to guide reflective thinking results in this kind of decision-making processes is unconsciously put on standby for a while. In the endeavour to continue or restart the decision-making process, technicians and/or the lean manager draws on the Lean A3 way of conducting root-cause analyses. Normally, it is necessary to cross disciplinary and/or organisational boundaries for gaining access to useful information and knowledge. This across disciplinary/organisation boundary collaboration is resource demanding and time-consuming. It other words, data and information are transferred back and forth disciplinary/organisational boundaries, which results in superfluous work for both Factory and external actors.

As for high complexity issues the consequences are partly perceptible at the shop floor in form of recurrent idling, slow running equipment and rescheduling of job orders. Workarounds implemented by technicians in collaboration with external actors mitigate the majority of the perceivable consequences at shop floors, but these are resource demanding. Likewise, the consequences of recurrent stock-out are not directly visible at the shop floor, but the ongoing rescheduling of purchase and job orders causes a lot of superfluous work for the administrative departments and managers at the shop floor. In general, the start-up of these decision-making processes has much in common with handling medium complexity issues. However, timewise the process is very protracted, from one to several years in which data, information and proposed technical solutions are handed back and forth disciplinary and organisational boundaries. These timeconsuming processes might be due to the facts that; i) Neither Factory nor the involved external actors have sufficient accessibility of data and information to guide reflective thinking and thus knowledge sharing towards implementing a proper solution, which results in the across boundary interactions are characterised by a trial and error approach. ii) The outcome of the decision making requires changes in organisational procedures including SFM procedures and in the technical equipment, which requires technicians to gain new experience.

\section{Discussion and conclusion}

The analysis of Factory demonstrates three kinds of decision-making processes in a smart manufacturing set-up. In general, because of a high degree of automation throughout the plant Factory has a high effective manufacturing.

This effectiveness characterises low complexity decision making, which normally are handled directly at the shop floor. Required data and information are directly accessible in the stand-alone OEE application or retrievable from log files embedded in the equipment, which are sufficient to guide practitioners reflective thinking. This promptly decision making means that; i) the hidden waste factors in form of obsolescent-, low accessibility-, scattered data/information and inappropriate coordination (see [22]) do not influence this kind of decision-making processes; ii) the consequences of the directly perceivable waste as scrap, idling and slow running equipment are reduced (see [21]); iii) the gap between actual time spend on handling the malfunction and necessary time is minimal, which means the amount of superfluous work is minimal.

Complex decision-making requires collaboration across professional and/or organisational boundaries. Hidden waste factors influence both medium and high decisionmaking complexity, but in different way. As for medium complexity issues, to guide 
reflective thinking various data and information are transferred back and forth disciplinary/organisational boundaries. The analysis indicates that it is resource-demanding to gain access to applicable data and information, and that the managerial focus is on handling the malfunction and thus accepting perceivable waste at the shop floor. Medium decision-making complexity is protracted and a gap between actual work and necessary work for handling the malfunction is apparent, which means the decisionmaking processes are characterised by superfluous work.

Handling highly complex issues are a lengthy process in which data and information are transferred back and forth disciplinary and organisational boundaries. Accessibility of data and information is a challenge, but the applicability of the scattered data/information seems even more challenging. In this regard, obsolescent-, low accessibility-, scattered data/information and inappropriate coordination lead to superfluous work. Because of the lengthy decision-making process, technicians in collaboration with external actors implement workarounds in the attempt to mitigate perceivable waste at the shop floors, but these workarounds are basically superfluous work. Thus, highly complexity decision making are characterised by two kind of superfluous work, transferring data and information back and forth boundaries and implementing workarounds.

The necessary work for accomplishing a decision-making process depends on the expe-rienced complexity. The necessary work is the minimum work if all involved practitio-ners have access to reliable data and can apply these to guide reflective thinking and knowledge sharing; often necessary work differs from the actual work invested in hand-ling the problematic situation. To gain an understanding of the difference between necessary and actual work this study suggest drawing on the principles of the lever-arm.

As illustrated in figure 1, the grey box on the left side of the lever-arm symbolises the actual work for handling a problematic issues. A part of the actual work consists of superfluous work, depicted above the dotted line. The grey box on the right side of the lever-arm illustrates the necessary work. As it appears from the figure, superfluous work causes disequilibrium between actual and necessary work. The requisite for gaining equilibrium is to move the fulcrum towards the actual work box. If the fulcrum falls directly in between the actual and necessary work boxes the decision-making processes are very effective; i.e., no superfluous work is done.

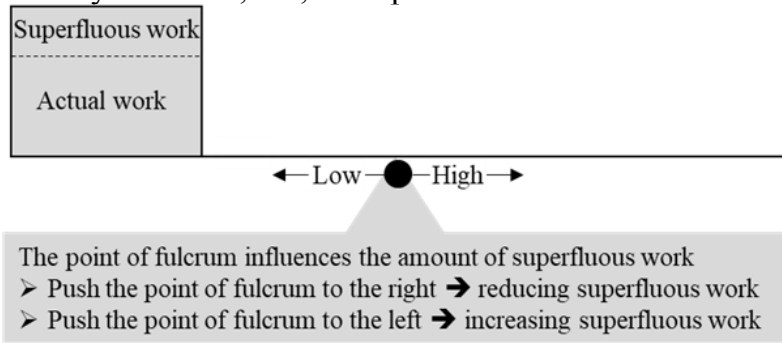

Figure 1. Superfluous work causes disequilibrium.

The placement of the fulcrum depicted at the lower part of figure 1 depends on the accessibility and applicability of data and information to guide reflective thinking and knowledge sharing when accomplishing a decision-making process. Low accessibility and applicability push the point of fulcrum to the left and thus increasing the amount of superfluous work, while high accessibility and applicability push the point of fulcrum to the right and thereby reducing superfluous work. 
As elaborated in the above, accessibility and applicability of data and information are the prerequisites for reducing superfluous work. Accessibility of data requires the formation of a reliable data architecture in terms of collecting, coding and storage data (see $[13 ; 24])$ and the establishment of sufficient interoperability among IT-systems to retrieve data (see $[10 ; 18 ; 23]$ ). However, the value of the accessible data depends solely on the information and knowledge sharing, which can be derived from the data. Reflective thinking is individualised (see [26]), but the practitioners' knowledge sharing does also involve social interactions. Knowledge sharing unfolds as a process of social interactions either within or across disciplinary/organisational boundaries in which the involved practitioners have "reflective conversation with" the accessible data (see [6]). In other words, accessibility and applicability are two side of the same coin; to reduce superfluous work managers should take into consideration both side of this coin.

Smart manufacturing is portrayed as an appropriate way to follow as it ensures an effective execution of the manufacturing processes and enables promptly date-driven decision making. This study demonstrates that the transition to the smart manufacturing set-up has enhanced the effectiveness of the manufacturing. However, the company being studied has not fused the flows of data/information, materials and digitalised equipment into CPS systems as suggested by Tao and Zhang [10], Qi et al. [11], eliminated information walls proposed by Dai et al. [13] and thereby enabled promptly data-driven decision making [2]. Actually, shop floor board meetings draw on analogue information written or printed on papers or whiteboards. Likewise, decision making crossing disciplinary/organisational boundaries are characterised by a lot of redundant work because of low accessibility of applicable data and information. These findings echo the viewpoint that practitioners involved in SFM decision making have not yet enjoyed the benefits of the current digitalisation of manufacturing [35]. Besides that practitioners do also face increasing decision-making complexity (like [16]) and managerial awareness for enhancing responsiveness (like [15]). Findings in this study indicate that the way to go for harvesting all benefits of smart manufacturing in terms of data-driven decision-making is still long and resource demanding. Apparently, companies involved in the transition towards a smart manufacturing set-up are successfully implementing an effective execution of manufacturing, but in blissful ignorance of the benefits of data-driven decision making. As this paper illustrates, the consequences are a lot of superfluous works at shop floors, across disciplinary and organisational boundaries.

\section{References}

[1] A. Kusiak, Smart manufacturing, International Journal of Production Research, 56:1-2, 2018

[2] D. Torres, C. Pimentel, and S. Duarte Shop floor management system in the context of smart manufacturing: a case study, Int. J. of Lean Six Sigma, 2019, in press.

[3] P. Ålstrom, and N. Modig, This is Lean: Resolving The Efficiency Paradox, Rheologica Pub., Halmstad, 2012

[4] J. Ma, F. Jiao, C.K. Lau, and Z. Lin, The relationships between shop floor management and QCCs to support Kaizen. International Journal of Quality \& Reliability Management, 35:9, 2018, Corpus ID: 56394940.

[5] C. Hertle, C. Siedelhofer, J. Metternich, E. Abele, Next generation shop floor management; how to continuously develop competencies in manufacturing environments. The 23rd Int. Conf. on Prod. Research, 2015

[6] J.B. Mathiasen and P. Clausen, Chasing digitised visualisation boards: revising the practices of decisionmaking at shop floor level, (forthcoming). 
[7] N. Wognum, C. Bil, F. Elgh, M. Peruzzini, J. Stjepandić, and W.J.C. Verhagen, Transdisciplinary systems engineering: implications, challenges and research agenda, International Journal of Agile Systems and Management, 12:1, 2019, pp. $58-89$.

[8] J.B. Mathiasen, and P. Clausen, Digitising Shop Floor Visualisation Boards: A Missing Link in the Industry 4.0 Era, Advances in Transdisciplinary Engineering, Vol. 10, 2019, pp. $189-198$.

[9] M. Holm, The future shop-floor operators, demands, requirements andinterpretations. Journal of Manufacturing Systems, 2018, Vol. 47, pp. 35-42.

[10] F. Tao, and M. Zhang, Digital Twin Shop-Floor: A New Shop-Floo, Special section of key technologies for smart manufacturing of industry 4.0, IEEE Access, 4:5, 2017, Corpus ID: 8600183.

[11] Q. Qi, D. Zhao, T.W. Liao, and F. Tao, Modeling of Cyber-Physical Systems and Digital Twin based on Edge Computing, Fog Computing and Cloud Computing towards Smart Manufacturing. Proceedings of the ASME 2018 13th International Manufacturing Science and Engineering Conference, 2018, DOI: 10.1115/MSEC2018-6435

[12] M.J. Mortenson, N.F. Doherty, and S. Robinson, Operational research from Taylorism to Terabytes: A research agenda for the analytics age, European Journal of Operational Research, 241, 2015, pp. 583-595.

[13] H.N. Dai, H. Wang, X. Guangquan, J. Wan and M. Imran, Big data analytics for manufacturing internet of things: opportunities, challenges and enabling technologies, Enterprise Information Systems, 2019, DOI: $10.1080 / 17517575.2019 .1633689$.

[14] Y. Yin, K. Stecke, and D. Li, The evolutionn of production systems from Industry 2.0 through Industry 4.0. Int. J. of Production Research, 2018, https://doi.org/10.1080/00207543.2017.1403664.

[15] L.G. Frank, L.S. Dalenogare, and N.F. Ayala, Industry 4.0 technologies: Implementation patterns in manufacturing companies, International Journal of Production Economics, 210, 2019, pp. 15-26.

[16] H. Kagermann, J. Helbig, A. Hellinger, and W. Wahlster, Securing the Future German Manufacturing Industry: Recommendations for Implementing the Strategic Initiative Industrie 4.0, acatec, 2013.

[17] K. Rajan, and A. Safiotti, Towards a science integrated of AI and Robotics, Artificial Intelligence, 2017, Vol. 247, pp. 1-9.

[18] B. Golzarpoor, C.T. Haas, D. Rayside, S. Kang, and M. Weston, Improving construction industry process interoperability with Industry Foundation Processes (IFP). Advanced Engineering Informatics, 38, 2018, pp. 555-568.

[19] C. Zhuang, J. Liu, and H. Xiong, Digital twin-based smart production management and control framework for the complex product assembly shop-floor, Int. J. of Advanced Manufacturing Technology, 2018, pp. 1149-1163.

[20] J.K. Liker, and D. Meier, The Toyota Way Fieldbook. McGraw-Hill, New York, 2006

[21] J.P. Womack, and D. Jones, Lean Thinking: Banish Waste and Create Wealth in Your Corporation, Simon and Schuster, London, 1996

[22] A. Sutrisno, I. Vanany, I. Gunawan, and M. Asjad, Lean waste classification model to support the sustainable operational practice, IOP Conference Series: Materials Science and Engineering, 2018

[23] L.Y. Pang, R.Y. Zhong, J. Fang, and G.Q. Huang, Data-source interoperability service for heterogeneous information integration in ubiquitous enterprises, Advanced Engineering Informatics, 29, 2015, pp. 549561.

[24] F. Tao, Q. Qi, A. Liu, and A. Kusiak, Data-driven smart manufacturing, J. of Manuf. Systems, 48, 2018, pp. 157-169.

[25] J.B. Mathiasen, and C. Koch, Product development as reading and writing doings within sociotechnical practices: the reciprocity between engineers and artefacts, Tech. Analysis \& Strategic Mgt. 27:5, 2015, pp. 604-620.

[26] U. Brandi, and B. Elkjær, Subtleties of knowledge sharing — Results from a case study within management consultancy, Knowledge Processes Management, 2019, pp. 185-193.

[27] J.B. Mathiasen, Learning within a product development working practice: An understanding anchored in pragmatism, Copenhagen 28, 2012

[28] J. Dewey, Logic: The Theory of Inquiry, Southern Illinois University Press, Carbondale, 1938

[29] C. Anderson, and P. Törnberg, Wickedness and the anatomy of complexity, Futures, 2018, pp. 118-138.

[30] S. Peng, L. Pelegrin, P.A. Luna, H. Horii and B.R. Moser, Problem Exploration with Bias Breaking Ideation for Maritime Emissions Reduction. Advances in Transdisciplinary Engineering, 10, pp.115-124.

[31] R.K. Yin, Case study research: Design and Methods, 3rd ed., Sage Publications, Thousand Oaks, 2003.

[32] A. Dubois, L.E. Gadde, Systematic combining: an abductive approach to case research. Journal of Business Research, 2002, pp. 553-560.

[33] R.E. Stake, The Case Study Method in Social Inquiry. In R. Gomm, M. Hammersley, and P. Foster, Case Study Method: Key Issues, Key Texts, SAGE Publications, London, 2000, pp. 19-26.

[34] A. Bryman, and E. Bell, Business Research Methods, $3^{\text {rd }}$ ed, Oxford University Press, Oxford, 2011.

[35] P. Clausen, and J.B. Mathiasen, Barriers and enablers for digitizing Shop Floor Management boards: Wireless Personal Communications. (Forthcoming) 